\title{
Best Speed Fit EDF Scheduling for Performance Asymmetric Multiprocessors
}

\author{
Peng $\mathrm{Wu}^{1}$ and Minsoo Ryu ${ }^{2}$ \\ ${ }^{1}$ Department of Electronics and Computer Engineering, Hanyang University, Seoul, Republic of Korea \\ ${ }^{2}$ Department of Computer Science and Engineering, Hanyang University, Seoul, Republic of Korea \\ Correspondence should be addressed to Minsoo Ryu; msryu@hanyang.ac.kr
}

Received 10 October 2016; Revised 19 January 2017; Accepted 1 February 2017; Published 20 February 2017

Academic Editor: Alessandro Gasparetto

Copyright (c) 2017 Peng Wu and Minsoo Ryu. This is an open access article distributed under the Creative Commons Attribution License, which permits unrestricted use, distribution, and reproduction in any medium, provided the original work is properly cited.

In order to improve the performance of a real-time system, asymmetric multiprocessors have been proposed. The benefits of improved system performance and reduced power consumption from such architectures cannot be fully exploited unless suitable task scheduling and task allocation approaches are implemented at the operating system level. Unfortunately, most of the previous research on scheduling algorithms for performance asymmetric multiprocessors is focused on task priority assignment. They simply assign the highest priority task to the fastest processor. In this paper, we propose BSF-EDF (best speed fit for earliest deadline first) for performance asymmetric multiprocessor scheduling. This approach chooses a suitable processor rather than the fastest one, when allocating tasks. With this proposed BSF-EDF scheduling, we also derive an effective schedulability test.

\section{Introduction}

Modern semiconductor technology adopts the use of multiple processors in their motherboard design because of the growing demand of performance and saving energy which cannot be handled by single processor systems. In this regard, performance asymmetric multiprocessors, where individual cores possess different performance, are believed to provide improved performance and low power consumption when compared to performance symmetric multiprocessors, where the cores are identical [1]. This is because such heterogeneous architecture allows the allocation of computing resources according to the needs of an application and better handling of dynamic workload requirements by exploiting speed of the fast cores and power efficiency of the slow cores available. One example of heterogeneous multiprocessor architectures is the ARM big.LITTLE architectures that combine slow and fast processing cores that possess identical instruction set but different execution speeds. The ARM big.LITTLE architectures are already used by Samsung in their Galaxy Note 5 and S6 Edge.

For embedded real-time systems, in addition to resource and power optimization, guaranteeing the deadlines of a realtime system is a critical requirement. Scheduling on such a performance asymmetric multicore platform is much more challenging than scheduling on identical multicore platform since the processing speed depends not only on the processor type, but also on the task executed. Thus, on heterogeneous multicore platforms, a decision that which type of processor will execute a given task over a period of time is also important.

Liu and Layland proposed earliest deadline first (EDF) scheduling algorithm on real-time single processor first in 1973 [2]. It is proved to be an optimal algorithm on single processor. Baker demonstrated an efficiently computable schedulability test for EDF scheduling on an asymmetric multiprocessors platform [3]. However, most of the previous research on EDF scheduling algorithm and relative scheduling algorithms for performance asymmetric multiprocessors is focused on the task priority approach [4-8]. They simply assign the highest priority task to the fastest processor. Since processors have different processing speeds in performance asymmetric multiprocessors, we believe that task allocation to an appropriate processor is also an important issue to be considered.

In the current research we focus on providing an improved scheduling algorithm based on EDF for handling 
real-time tasks for performance asymmetric multiprocessor systems. More specifically, we propose a new EDF scheduling algorithm, best speed fit EDF (BSF-EDF). For BSF-EDF, the priorities of the tasks are chosen according to the original EDF scheduling algorithm, whereas it differs in terms of task allocation to processors, such that in the former approach the highest priority task is assigned to a faster processor and in the latter approach the highest priority task is allocated to the appropriate idle processor. We also obtain a new schedulability test for the BSF-EDF on performance asymmetric multiprocessors.

The rest of the paper is organized as follows. In Section 2, we describe system model and background. Section 3 presents the BSF-EDF scheduling with an example. In Section 4, we describe the proposed schedulability test for the BSF-EDF scheduling algorithm.

\section{System Model and Background}

We consider an $m$-core performance asymmetric multiprocessor system and we use $\pi$ to denote the set of $m$ performance asymmetric processors. We use $s_{i}$ to represent the processing speed of any processor $P_{i}$. For convenience we use 1 to represent the speed of the slowest processor, such that the speed of faster processors can be represented by a multiple of 1 ; for example, $s_{i}=3$. We also assume that the speeds of processors are specified in nondecreasing order: $s_{i} \leq s_{i+1}$ for all $i$. Let $S_{i}$ be the cumulative sum of processor speeds of the first $i$ processors in a performance asymmetric multiprocessors platform $\pi$ :

$$
S_{i}=\sum_{j=1}^{i} s_{j} .
$$

Immediately, $S_{m}$ denotes the cumulative sum of $\pi$.

In this paper, we consider a sporadic task model. Let $\Gamma=\left\{\tau_{1}, \tau_{2}, \tau_{3}, \ldots, \tau_{n}\right\}$ be a set of $n$ sporadic tasks. A sporadic task $\tau_{i}$ is defined by a 3-tuple $\left(e_{i}, d_{i}, T_{i}\right)$, where $e_{i}$ is the worst case execution time when it is executed in the lowest speed processor, $d_{i}$ is the relative constrained deadline such that $e_{i} \leq d_{i} \leq T_{i}$, and $T_{i}$ is the minimum interarrival time, which is also referred as period of the task. The minimum interarrival time $T_{i}$ means that any two consecutive requests of $\tau_{i}$ are separated by at least $T_{i}$. We use $\tau_{i, j}$ to represent the $j$ th job of task $\tau_{i}$ and $A_{i, j}$ to denote the arrival time of $\tau_{i, j}$. Note that a task can have different execution time based on different speeds of processors. Therefore, a job executing on the $j$ th processor for a duration of $t$ time can receive $t \times s_{j}$ units of execution.

Now we introduce several further notions that we will use for schedulability analysis. Since processors have varying speeds, we use the slowest processor to measure the worst case execution time $e_{i}$ of a task $\tau_{i}$. The utilization $u_{i}$ of a task $\tau_{i}$ is the ratio of the worst case execution time to its period, that is, $e_{i} / T_{i}$. The total utilization $U_{\text {sum }}(\Gamma)$ and the largest task utilization $u_{\max }(\Gamma)$ of a task set are defined as follows:

$$
\begin{aligned}
& U_{\text {sum }}(\Gamma)=\sum_{\tau_{i} \in \Gamma} u_{i}, \\
& u_{\max }(\Gamma)=\max _{\tau_{i} \in \Gamma} u_{i} .
\end{aligned}
$$

The density $\delta_{i}$ of a task $\tau_{i}$ is defined to be the ratio of $e_{i} / d_{i}$, that is, ratio of the worst case execution time to the relative deadline. The maximum density $\delta_{\max }(\Gamma)$ of a task set $\Gamma$ is defined as follows:

$$
\delta_{\max }(\Gamma)=\max _{\tau_{i} \in \Gamma} \delta_{i}
$$

The concepts of processor demand bound function $\operatorname{DBF}\left(\tau_{i}, \Delta t\right)$ and processor load are used in the analysis of multiprocessor scheduling and are well-studied in detail in [9]. $\operatorname{DBF}\left(\tau_{i}, \Delta t\right)$ is used to represent an upper bound on the maximum cumulative processing time demand by jobs of $\tau_{i}$ that arrive in and also have deadline within any interval of length $\Delta t$. It has been shown that

$$
\begin{aligned}
& \operatorname{DBF}\left(\tau_{i}, \Delta t\right) \\
& \stackrel{\text { def }}{=} \begin{cases}\max \left(0,\left(\left\lfloor\frac{\Delta t-d_{i}}{T_{i}}\right\rfloor+1\right) e_{i}\right) & \text { if } \Delta t \geq d_{i} \\
e_{i} & \text { if } \Delta t<d_{i} .\end{cases}
\end{aligned}
$$

The processor load parameter is based on the DBF function which is the maximum value of the ratio of processor demand bound and length of the time interval $\Delta t[10]$.

$$
\operatorname{LOAD}(\Gamma) \stackrel{\text { def }}{=} \max _{\Delta t>0}\left(\frac{\sum_{\tau_{i} \in \Gamma} \operatorname{DBF}\left(\tau_{i}, \Delta t\right)}{\Delta t}\right) .
$$

\section{BSF-EDF Scheduling Algorithm for Performance Asymmetric Multiprocessor Platform}

Our scheduling algorithm proposed in this paper is a priority driven scheduling algorithm, which means that the highest priority task always runs first. Moreover, we choose global multiprocessor scheduling policy. Global scheduling maintains a single system-wide queue of ready tasks, from which tasks are extracted at run-time, to be scheduled on the available computing resources. Global scheduling is more appropriate for open systems, as there is no need to run load balancing algorithms when the set of tasks changes. We also allow preemption of tasks in our proposed BSFEDF scheduling algorithm and migration of tasks between different speed processors is also permitted.

Now there are two crucial issues to be addressed, task priority assignment and task allocation to processors. For BSF-EDF scheduling we assign the priorities to tasks according to their absolute deadline, such that the task with earlier deadline has higher priority. In previous research work, individuals simply assign the highest priority task to the fastest idle processor. Contrary to this, we assign the highest priority task to the appropriate idle processor rather than the fastest idle one. The appropriate processor is the slowest speed processor which ensures that a task can be executed without missing its deadline. In other words, we choose the slowest speed idle processor whose speed is no less than the utilization of the task. If there does not exist such appropriate processor, we allocate the task to the slowest idle processor. We should reschedule all the tasks whenever a schedule event 


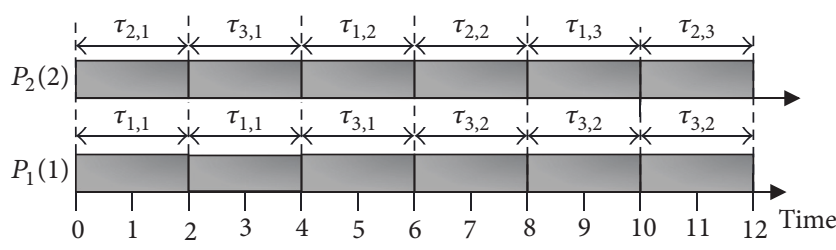

FIGURE 1: Task schedule under BSF-EDF.

occurs. Schedule events include two cases, when (a) a running job is finished and (b) a new job has arrived. Specifically, we say that (1) no processor is idle while there is an active task, (2) a scheduled task always executes on the best speed fit processor, if there exists such a processor, and (3) higher priority task always runs first.

Example 1. Figure 1 manifests a simple BSF-EDF scheduling example on a performance asymmetric multiprocessor platform. Consider a performance asymmetric multiprocessor platform $\pi=\{1,2\}$ which has two processors and a task set $\Gamma=\left\{\tau_{1}(4,4,4), \tau_{2}(4,4,4), \tau_{3}(6,6,6)\right\}$. Initially, $\tau_{1,1}$ has the highest priority, and $P_{1}$ is the appropriate idle processor, so we allocate it to $P_{1}$. The second priority job $\tau_{2,1}$ is allocated to processor $P_{2}$. Job $\tau_{2,1}$ finishes at time $t=2$; therefore, time $t=2$ is a schedule event time. All tasks should be rescheduled. $\tau_{1,1}$ still has the highest priority; we allocate it to $P_{1}$, and we allocate second priority job $\tau_{3,1}$ to $P_{2} . \tau_{1,1}$ finishes its execution at time $t=4$, so a schedule event appears. Now $\tau_{3,1}$ has the highest priority, so we allocate it to $P_{1}$, and we allocate job $\tau_{1,2}$ to $P_{2}$. It is easy to see that all tasks will satisfy their deadline under BSF-EDF. The processors are used to their $100 \%$ utilization. As shown in the figure, $t=\{2,4,6,8,10,12\}$ are schedule event points.

\section{BSF-EDF Schedulability Analysis for Performance Asymmetric Multiprocessor Platform}

With respect to a given platform, a given sporadic task set is said to be BSF-EDF schedulable if the schedule is able to meet all deadlines for every collection of jobs that may be generated by the task set. In this section, we derive a schedulability test for the BSF-EDF scheduling on performance asymmetric multiprocessors.

We first obtain a necessary condition when a task misses its deadline for the BSF-EDF scheduling. By taking the contrapositive of the condition, we obtain a sufficient schedulability condition.

Suppose that a job $\tau_{i, j}$ of task $\tau_{i}$ is the one to first miss its deadline at time-instant $A_{i, j}+d_{i}$ (see Figure 2). $A_{i, j}$ denotes the arrival time of job $\tau_{i, j}$. Due to the properties of BSFEDF scheduling, we discard the legal sequence of jobs with deadlines later than $A_{i, j}+d_{i}$. We only consider the BSFEDF schedule of the remaining legal sequence of job requests, since the later deadline jobs have no effect on the scheduling of earlier deadline ones under BSF-EDF. It follows that a

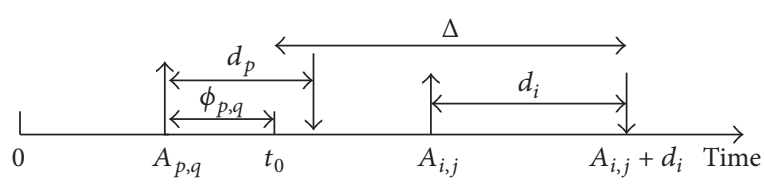

FIgURe 2: A job $\tau_{i, j}$ of task $\tau_{i}$ arrives at $A_{i, j}$ and misses its deadline at time-instant $A_{i, j}+d_{i}$.

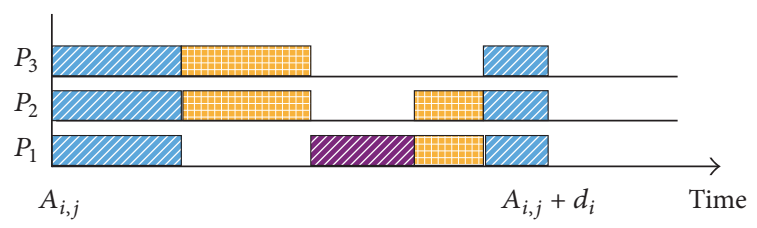

FIGURE 3: An example: there are three processors, and the total length of blue color units is $B_{3}$. The total length of orange color units is $B_{2}$. The total length of purple color units is $B_{1}$.

deadline miss of $\tau_{i}$ occurs at time-instant $A_{i, j}+d_{i}$ (and this is the earliest deadline miss).

For a given task set $\Gamma=\left\{\tau_{1}, \tau_{2}, \tau_{3}, \ldots, \tau_{n}\right\}$, let $W\left(t_{p}, t_{q}\right)$ be the total amount of work done during $\left[t_{p}, t_{q}\right)$, and let $R\left(t_{p}, t_{q}\right)$ be the total amount of processor time demand in $\left[t_{p}, t_{q}\right)$ required by all the jobs in this legal sequence of task set $\Gamma$.

We derive a necessary condition for a task $\tau_{i}$ to miss its deadline, by following three steps: (1) deriving a lower bound on the total amount of processor time demand during the time interval $\left[t_{0}, A_{i, j}+d_{i}\right.$ ) in Section 4.1, (2) deriving an upper bound on the total amount of processor time demand during the time interval $\left[t_{0}, A_{i, j}+d_{i}\right)$ in Section 4.2, and (3) combining the lower bound and upper bound into a necessary condition for task $\tau_{i}$ missing its deadline. Note that $t_{0}$ is a special time point that will be explained in the coming section.

4.1. Lower Bound. In this section, we obtain a lower bound of $R\left(t_{0}, A_{i, j}+d_{i}\right)$.

Consider job $\tau_{i, j}$ arriving at time $A_{i, j}$ misses its deadline at time-instant $A_{i, j}+d_{i}$. Let $B_{v}$ be the total duration over $\left[A_{i, j}, A_{i, j}+d_{i}\right)$ for which exactly $v$ processors are busy in this BFS-EDF schedule, $0 \leq v \leq m$. Note that $B_{0}$ is necessarily zero, since $\tau_{i, j}$ does not finish before its deadline. Figure 3 manifests an example to understand this definition. $S_{v}^{\prime}$ denotes the cumulative speed of the currently running $v$ processors. We can then write

$$
W\left(A_{i, j}, A_{i, j}+d_{i}\right)=\sum_{v=1}^{m} S_{v}^{\prime} B_{v} .
$$

Since $\sum_{v=1}^{m} S_{v}^{\prime} B_{v}=S_{m} B_{m}+\sum_{v=1}^{m-1} S_{v}^{\prime} B_{v}$ and $S_{m} B_{m}=S_{m}\left(d_{i}-\right.$ $\sum_{v=1}^{m-1} B_{v}$ ), we can write

$$
W\left(A_{i, j}, A_{i, j}+d_{i}\right)=S_{m} d_{i}-\sum_{v=1}^{m-1}\left(S_{m} B_{v}-S_{v}^{\prime} B_{v}\right) .
$$

As we mentioned in Section 2, $S_{v}$ means the cumulative speed of the first $v$ lowest speed processors. The tasks may 
be allocated to the faster speed processors under BSF-EDF; therefore we can get

$$
S_{v}^{\prime} \geq S_{v} .
$$

From (7) and (8) above, we conclude that

$$
\begin{aligned}
W\left(A_{i, j}, A_{i, j}+d_{i}\right) & \geq S_{m} d_{i}-\sum_{v=1}^{m-1}\left(S_{m} B_{v}-S_{v} B_{v}\right) \\
& =S_{m} d_{i}-\sum_{v=1}^{m-1} \frac{S_{m}-S_{v}}{s_{1}} s_{1} B_{v} .
\end{aligned}
$$

Let $\lambda(\pi)=\max _{1 \leq i \leq m}\left(\sum_{j=i+1}^{m} s_{j} / s_{1}\right)$. It immediately follows that

$$
\lambda(\pi) \geq \frac{S_{m}-S_{v}}{s_{1}} \quad \text { for } 1 \leq v \leq m-1 .
$$

Combining (9) and (10), we have

$$
W\left(A_{i, j}, A_{i, j}+d_{i}\right) \geq S_{m} d_{i}-\lambda(\pi) \sum_{v=1}^{m-1} s_{1} B_{v} .
$$

Due to the work conserving property of BSF-EDF, $\tau_{i, j}$ must always be running in one of processors in time interval $\left[A_{i, j}, A_{i, j}+d_{i}\right)$, except when all the processors are busy. The processor time that $\tau_{i, j}$ receives during $\left[A_{i, j}, A_{i, j}+d_{i}\right)$ cannot be less than $\sum_{v=1}^{m-1} s_{1} B_{v}$, which is the worst case processor time when $\tau_{i, j}$ is running on the slowest processor $s_{1}$. The processor time that $\tau_{i, j}$ receives must not exceed $e_{i}$, since job $\tau_{i, j}$ misses its deadline. Thus, we have

$$
e_{i}>\sum_{v=1}^{m-1} s_{1} B_{v}
$$

By applying this to (11), we have

$$
\begin{aligned}
& W\left(A_{i, j}, A_{i, j}+d_{i}\right)>S_{m} d_{i}-\lambda(\pi) e_{i}, \\
& \frac{W\left(A_{i, j}, A_{i, j}+d_{i}\right)}{d_{i}}>S_{m}-\lambda(\pi) \delta_{i} .
\end{aligned}
$$

Since $\delta_{\max }(\Gamma) \geq \delta_{i}$, we can write

$$
\frac{W\left(A_{i, j}, A_{i, j}+d_{i}\right)}{d_{i}}>S_{m}-\lambda(\pi) \delta_{\max }(\Gamma) .
$$

Let

$$
\mu=S_{m}-\lambda(\pi) \delta_{\max }(\Gamma)
$$

Then we have

$$
W\left(A_{i, j}, A_{i, j}+d_{i}\right)>\mu d_{i} .
$$

Based on this, we now obtain a lower bound of $R\left(t_{0}, A_{i, j}+d_{i}\right)$, where $t_{0}$ is the earliest time point $t$ such that $t \leq A_{i, j}$ and $W\left(t, A_{i, j}+d_{i}\right)>\mu\left(A_{i, j}+d_{i}-t\right)$. Note that we can always find such a time instant since at least $A_{i, j}$ satisfied $A_{i, j} \leq A_{i, j}$ and $W\left(A_{i, j}, A_{i, j}+d_{i}\right)>\mu\left(A_{i, j}+d_{i}-A_{i, j}\right)$. Let $\Delta=A_{i, j}+d_{i}-t_{0}$; then we finally have

$$
W\left(t_{0}, A_{i, j}+d_{i}\right)>\mu \Delta .
$$

Note that the job $\tau_{i, j}$ cannot be completed before time $A_{i, j}+d_{i}$, so the total amount of work processed can never exceed the amount of processor time demand. Thus, we have

$$
R\left(t_{0}, A_{i, j}+d_{i}\right) \geq W\left(t_{0}, A_{i, j}+d_{i}\right) .
$$

It immediately follows that

$$
R\left(t_{0}, A_{i, j}+d_{i}\right)>\mu \Delta .
$$

As a result, $\mu \Delta$ is a lower bound of the total amount of processor time demand during the time interval $\left[t_{0}, A_{i, j}+d_{i}\right)$.

4.2. Upper Bound. We now obtain an upper bound of $R\left(t_{0}, A_{i, j}+d_{i}\right)$ of the total amount of processor time demand. For this, we need to consider two types of jobs, regular jobs that arrived at or after $t_{0}$ and the jobs that arrive before $t_{0}$ but have not completed execution in the BSF-EDF schedule by the time-instant $t_{0}$. We will refer to jobs arriving prior to $t_{0}$ that need execution over $\left[t_{0}, A_{i, j}+d_{i}\right)$ as carry-in jobs. We use $R_{c}$ to denote the total amount of processor time demand caused by carry-in jobs and use $R_{r}$ to denote the total amount of processor time demand caused by the regular jobs.

We first compute the upper bound of $R_{c}$ by (1) determining an upper bound on the remaining processor time demand for each carry-in job, (2) determining a maximum value of the number of carry-in jobs, and (3) combining the per-job bounds to obtain an upper bound on the total remaining processor time demand of all carry-in jobs.

Lemma 2. Each carry-in job has less than $\delta_{\max }(\Gamma) \Delta$ remaining execution requirement at time-instant $t_{0}$.

Proof. Let us consider a carry-in job $\tau_{p, q}$ of a task $\tau_{p}$ that arrives at time-instant $A_{p, q}<t_{0}$ and has not completed its execution by time $t_{0}$ (see Figure 2). Let $\phi_{p, q}=t_{0}-A_{p, q}$. Let $e_{p, q}^{\prime}$ be the processing time that $\tau_{p, q}$ receives during time interval $\left[A_{p, q}, t_{0}\right)$. By using the same approach as in Section 4.1, let $I_{v}$ be the total duration over $\left[A_{p, q}, t_{0}\right)$ for which exactly $v$ processors are busy in this BSF-EDF schedule. $J_{v}$ denotes the cumulative speed of the currently running $v$ processors over $\left[A_{p, q}, t_{0}\right)$. Thus, the total amount of work processed in $\left[A_{p, q}, t_{0}\right)$ can be calculated by $S_{m} \phi_{p, q}-\sum_{v=1}^{m-1}\left(S_{m} I_{v}-J_{v} I_{v}\right)$. Since $J_{v} \geq S_{v}$, it immediately follows that

$$
e_{p, q}^{\prime} \geq S_{m} \phi_{p, q}-\sum_{v=1}^{m-1}\left(S_{m} I_{v}-S_{v} I_{v}\right)
$$

Since we can also express the total amount of work done during the time interval $\left[A_{p, q}, t_{0}\right)$ by $W\left(A_{p, q}, A_{i, j}+d_{i}\right)-$ $W\left(t_{0}, A_{i, j}+d_{i}\right)$, we can write

$$
W\left(A_{p, q}, A_{i, j}+d_{i}\right)-W\left(t_{0}, A_{i, j}+d_{i}\right)=e_{p, q}^{\prime} .
$$


By applying (20) to (21), we have

$$
\begin{gathered}
W\left(A_{p, q}, A_{i, j}+d_{i}\right)-W\left(t_{0}, A_{i, j}+d_{i}\right) \\
\geq S_{m} \phi_{p, q}-\sum_{v=1}^{m-1}\left(S_{m} I_{v}-S_{v} I_{v}\right) .
\end{gathered}
$$

By the definition of $t_{0}$ (see (17)), it must satisfy

$$
W\left(t_{0}, A_{i, j}+d_{i}\right)>\mu \Delta .
$$

Note that $t_{0}$ is the earliest time point $t$ such that $t \leq A_{i, j}$ and $W\left(t, A_{i, j}+d_{i}\right)>\mu\left(A_{i, j}+d_{i}-t\right)$, and we can also get

$$
W\left(A_{p, q}, A_{i, j}+d_{i}\right) \leq \mu\left(\Delta+\phi_{p, q}\right) .
$$

It immediately follows that

$$
\begin{aligned}
\mu\left(\Delta+\phi_{p, q}\right)-\mu \Delta> & W\left(A_{p, q}, A_{i, j}+d_{i}\right) \\
& -W\left(t_{0}, A_{i, j}+d_{i}\right) .
\end{aligned}
$$

By combining it to (22), we have

$$
\begin{gathered}
\mu\left(\Delta+\phi_{p, q}\right)-\mu \Delta>S_{m} \phi_{p, q}-\sum_{v=1}^{m-1}\left(S_{m} I_{v}-S_{v} I_{v}\right) \Longrightarrow \\
\mu \phi_{p, q}>S_{m} \phi_{p, q}-\sum_{v=1}^{m-1}\left(S_{m} I_{v}-S_{v} I_{v}\right) \Longrightarrow \\
\mu \phi_{p, q}>S_{m} \phi_{p, q}-\sum_{v=1}^{m-1} \frac{S_{m}-S_{v}}{s_{1}} s_{1} I_{v} .
\end{gathered}
$$

Since

$$
\begin{aligned}
& \lambda(\pi)=\max _{1 \leq i \leq m} \frac{\sum_{j=i+1}^{m} s_{j}}{s_{1}}, \\
& \lambda(\pi) \geq \frac{S_{m}-S_{v}}{s_{1}} \text { for } 1 \leq v \leq m-1,
\end{aligned}
$$

we can write

$$
S_{m} \phi_{p, q}-\sum_{v=1}^{m-1} \frac{S_{m}-S_{v}}{s_{1}} s_{1} I_{v} \geq S_{m} \phi_{p, q}-\lambda(\pi) \sum_{v=1}^{m-1} s_{1} I_{v} .
$$

Equation (26) then becomes

$$
\mu \phi_{p, q}>S_{m} \phi_{p, q}-\lambda(\pi) \sum_{v=1}^{m-1} s_{1} I_{v} .
$$

Observe that the amount of execution that carry-in job $\tau_{p, q}$ receives over $\left[A_{p, q}, t_{0}\right)$ is no less than $\sum_{v=1}^{m-1} I_{v} s_{1}$ since $\tau_{p, q}$ must be executing on one of the processors during any instant, when any processor is idle. Thus, we have

$$
e_{p, q}^{\prime} \geq \sum_{v=1}^{m-1} s_{1} I_{v}
$$

It immediately follows that

$$
S_{m} \phi_{p, q}-\lambda(\pi) \sum_{v=1}^{m-1} s_{1} I_{v} \geq S_{m} \phi_{p, q}-\lambda(\pi) e_{p, q}^{\prime} .
$$

By (29) and (31), we can write

$$
\mu \phi_{p, q}>S_{m} \phi_{p, q}-\lambda(\pi) e_{p, q}^{\prime} .
$$

Using $\mu=S_{m}-\lambda(\pi) \delta_{\max }(\Gamma)$ (defined in Section 4.1) in (32), it follows that

$$
\left(S_{m}-\lambda(\pi) \delta_{\max }(\Gamma)\right) \phi_{p, q}>S_{m} \phi_{p, q}-\lambda(\pi) e_{p, q}^{\prime},
$$

resulting in

$$
e_{p, q}^{\prime}>\delta_{\max }(\Gamma) \phi_{p, q} .
$$

Note that $e_{p, q}=d_{p} \delta_{p}$ and that the remaining processing time demand of the carry-in job $\tau_{p, q}$ of task is $e_{p, q}-e_{p, q}^{\prime}$. Thus, we have

$$
e_{p, q}-e_{p, q}^{\prime}<d_{p} \delta_{p}-\delta_{\max }(\Gamma) \phi_{p, q} .
$$

The absolute deadline of carry-in job $\tau_{p, q}$ is no later than time $A_{i, j}+d_{i}$; that is, $d_{p}-\phi_{p, q} \leq \Delta$. Also, $\delta_{p} \leq \delta_{\max }(\Gamma)$; therefore

$$
\begin{aligned}
& e_{p, q}-e_{p, q}^{\prime}<d_{p} \delta_{p}-\delta_{\max }(\Gamma) \phi_{p, q}, \\
& e_{p, q}-e_{p, q}^{\prime}<d_{p} \delta_{\max }(\Gamma)-\delta_{\max }(\Gamma) \phi_{p, q} \\
& e_{p, q}-e_{p, q}^{\prime}<\delta_{\max }(\Gamma)\left(d_{p}-\phi_{p, q}\right) \\
& e_{p, q}-e_{p, q}^{\prime}<\delta_{\max }(\Gamma) \Delta .
\end{aligned}
$$

As a result, an upper bound of the remaining processor time demand for each carry-in job can be expressed as $\delta_{\max }(\Gamma) \Delta$.

We obtained the upper bound on the remaining processor time demand for each carry-in job. We will now determine the maximum value of the number of carry-in jobs.

Lemma 3. The number of carry-in jobs is bounded by

$$
\Omega=\max \left\{\omega: S_{\omega}<\mu\right\} .
$$

Proof. Let $\varepsilon$ be an arbitrarily small positive number. From the definition of $t_{0}$, it follows that

$$
\begin{gathered}
W\left(t_{0}, A_{i, j}+d_{i}\right)>\mu \Delta, \\
W\left(t_{0}-\varepsilon, A_{i, j}+d_{i}\right) \leq \mu(\varepsilon+\Delta) .
\end{gathered}
$$

Therefore, the work processed during $\left[t_{0}-\varepsilon, t_{0}\right)$ is less than $\mu \varepsilon$ since $W\left(t_{0}-\varepsilon, A_{i, j}+d_{i}\right)-W\left(t_{0}, A_{i, j}+d_{i}\right)<\mu \varepsilon$. Based on the definition of $\mu \leq S_{m}$, it follows that the work processed during $\left[t_{0}-\varepsilon, t_{0}\right)$ is less than $\varepsilon S_{m}$. Therefore some processor is idle during $\left[t_{0}-\varepsilon, t_{0}\right)$. This implies that all carry-in jobs 
are running in this interval and the number of carry-in jobs is the same as the number of busy processors. Let $\omega$ be the number of busy processors over $\left[t_{0}-\varepsilon, t_{0}\right)$ and let $\Omega$ be an upper bound of $\omega$. Thus, we have

$$
\Omega=\max \left\{\omega: S_{\omega}<\mu\right\} .
$$

Therefore the upper bound of $R_{c}$ is $\Omega \Delta \delta_{\max }(\Gamma)$.

Now let us consider the upper bound of the total amount of processor time demand caused by regular jobs. Fortunately, it is a well-studied subject, and algorithms are known for computing DBF and $\operatorname{LOAD}[9,10]$. Baruah et al. showed that the upper bound of $R_{r}$ can be calculated by $\Delta \times \operatorname{LOAD}(\Gamma)$, where

$$
\begin{aligned}
& \operatorname{LOAD}(\Gamma) \stackrel{\text { def }}{=} \max _{\Delta t>0}\left(\frac{\sum_{\tau_{i} \in \Gamma} \operatorname{DBF}\left(\tau_{i}, \Delta t\right)}{\Delta t}\right), \\
& \operatorname{DBF}\left(\tau_{i}, \Delta t\right) \\
& \quad \stackrel{\text { def }}{=} \begin{cases}\max \left(0,\left(\left\lfloor\frac{\Delta t-d_{i}}{T_{i}}\right\rfloor+1\right) e_{i}\right) & \text { if } \Delta t \geq d_{i} \\
e_{i} & \text { if } \Delta t<d_{i} .\end{cases}
\end{aligned}
$$

As a result, an upper bound of the total amount of processor time demand can be expressed as

$$
R\left(t_{0}, A_{i, j}+d_{i}\right) \leq \operatorname{LOAD}(\Gamma) \Delta+\Omega \Delta \delta_{\max }(\Gamma)
$$

4.3. BSF-EDF Schedulability Test. We now describe a sufficient schedulability test for the BSF-EDF scheduling algorithm.

Theorem 4. A task set $\Gamma=\left\{\tau_{1}, \tau_{2}, \tau_{3}, \ldots, \tau_{n}\right\}$ is schedulable by BSF-EDF on an $m$-core performance asymmetric multiprocessor platform $\pi=\left\{P_{1}, P_{2}, \ldots, P_{m}\right\}$, with processing speeds $s_{1}=s_{2}=\cdots=s_{q}=1$, and $s_{i}>1$ for $q<i \leq m$, if it satisfies the following condition:

$$
\operatorname{LOAD}(\Gamma) \leq \mu-\Omega \delta_{\max }(\Gamma)
$$

where $\mu$ and $\Omega$ are defined in (15) and (37), respectively.

Proof. We prove it through the principle of contradiction. We obtain necessary condition for the job $\tau_{i, j}$ missing its deadline. Negating this condition yields a sufficient condition for the BSF-EDF schedulability.

From the foregoing discussion, we already got the upper bound and lower bound of the total amount of processor time demand during the time interval $\left[t_{0}, A_{i, j}+d_{i}\right)$. As a result, we obtain the following bound on $R\left(t_{0}, A_{i, j}+d_{i}\right)$ :

$$
\begin{aligned}
& R\left(t_{0}, A_{i, j}+d_{i}\right) \leq \operatorname{LOAD}(\Gamma) \Delta+\Omega \Delta \delta_{\max }(\Gamma), \\
& R\left(t_{0}, A_{i, j}+d_{i}\right)>\mu \Delta .
\end{aligned}
$$

Based on (43), we can get the necessary condition for a task $\tau_{i}$ to miss its deadline:

$$
\begin{gathered}
\operatorname{LOAD}(\Gamma) \Delta+\Omega \Delta \delta_{\max }(\Gamma)>\mu \Delta \\
\equiv \operatorname{LOAD}(\Gamma)+\Omega \delta_{\max }(\Gamma)>\mu \\
\equiv \operatorname{LOAD}(\Gamma)>\mu-\Omega \delta_{\max }(\Gamma) .
\end{gathered}
$$

Equivalently, the negation of this condition is sufficient to ensure BSF-EDF schedulability:

$$
\operatorname{LOAD}(\Gamma) \leq \mu-\Omega \delta_{\max }(\Gamma)
$$

which is as claimed in Theorem 4 .

\section{Conclusions}

In this paper, we presented a unique approach to task allocation in a performance asymmetric multiprocessor system based on EDF, called BSF-EDF. In BSF-EDF we chose the appropriate slowest speed processor to execute the highest priority task. To our knowledge, this is the first piece of work that considers the task allocation problem on performance asymmetric multiprocessors. We hold the view that choosing an appropriate processor instead of a fastest one gives opportunities of improved scheduling, by reserving fast processors for future tasks that will arrive later. We have also presented an effective schedulability test for performance asymmetric multiprocessors based on the properties of the proposed BSFEDF.

\section{Competing Interests}

The authors declare that there is no conflict of interests regarding the publication of this paper.

\section{Acknowledgments}

This work was supported partly by the ICT R\&D Program of MSIP/IITP [R01141600460001002, Software Black Box for Highly Dependable Computing] and partly by the National Research Foundation of Korea (NRF) Grant funded by the Korean Government (MSIP) (NRF-2015R1A5A7037751).

\section{References}

[1] T. Y. Morad, U. C. Weiser, A. Kolodny, M. Valero, and E. Ayguadé, "Performance, power efficiency and scalability of asymmetric cluster chip multiprocessors," IEEE Computer Architecture Letters, vol. 5, no. 1, pp. 14-17, 2006.

[2] C. L. Liu and J. W. Layland, "Scheduling algorithms for multiprogramming in a hard-real-time environment," Journal of the Association for Computing Machinery, vol. 20, pp. 46-61, 1973.

[3] T. P. Baker, "Multiprocessor EDF and deadline monotonic schedulability analysis," in Proceedings of the 24th IEEE International Real-Time Systems Symposium (RTSS '03), pp. 120-129, Washington, DC, USA, December 2003.

[4] J. Lee and I. Shin, "EDZL schedulability analysis in realtime multicore scheduling," IEEE Transactions on Software Engineering, vol. 39, no. 7, pp. 910-916, 2013. 
[5] M. Cirinei and T. P. Baker, "EDZL scheduling analysis," in Proceedings of the 19th Euromicro Conference on Real-Time Systems (ECRTS '07), pp. 9-18, Pisa, Italy, July 2007.

[6] H. W. Wei, Y. H. Chao, S. S. Lin, K. J. Lin, and W. K. Shih, "Current results on EDZL scheduling for multiprocessor realtime systems," in Proceedings of the 13th IEEE International Conference on Embedded and Real-Time Computing Systems and Applications (RTCSA '07), pp. 120-130, 2007.

[7] Y.-H. Chao, S.-S. Lin, and K.-J. Lin, "Schedulability issues for EDZL scheduling on real-time multiprocessor systems," Information Processing Letters, vol. 107, no. 5, pp. 158-164, 2008.

[8] P. Wu and M. Ryu, "EDZL scheduling and schedulability analysis for performance asymmetric multiprocessors," International Journal of Foundations of Computer Science, vol. 27, no. 1, pp. 1-14, 2016.

[9] S. K. Baruah, A. K. Mok, and L. E. Rosier, "Preemptively scheduling hard-real-time sporadic tasks on one processor," in Proceedings of the 11th Real-Time Systems Symposium (RTSS '90), pp. 182-190, IEEE, Lake Buena Vista, Fla, USA, December 1990.

[10] T. P. Baker, N. Fisher, and S. Baruah, "Algorithms for determining the load of a sporadic task system," Tech. Rep. TR-051201, 2005. 


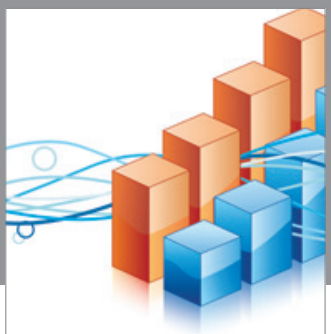

Advances in

Operations Research

vatem alat4

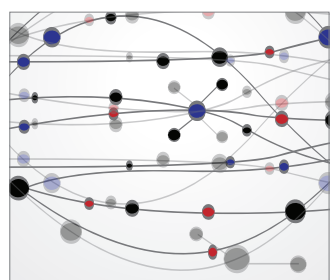

\section{The Scientific} World Journal
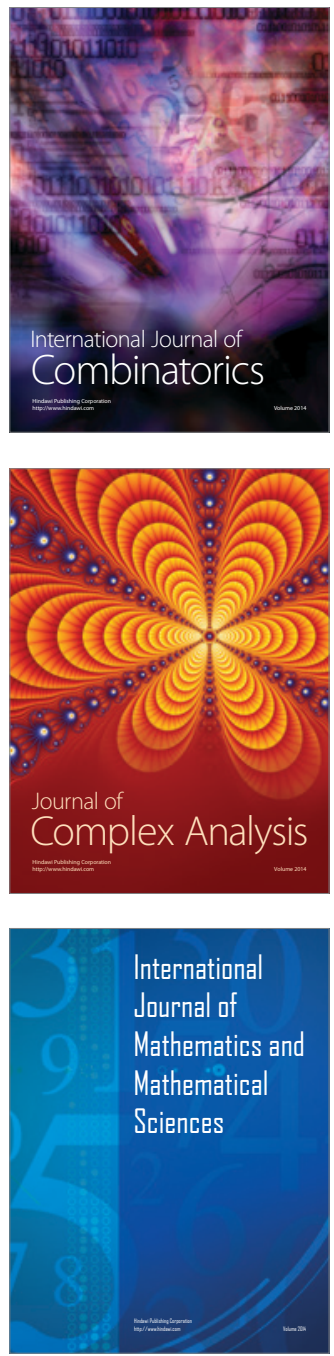
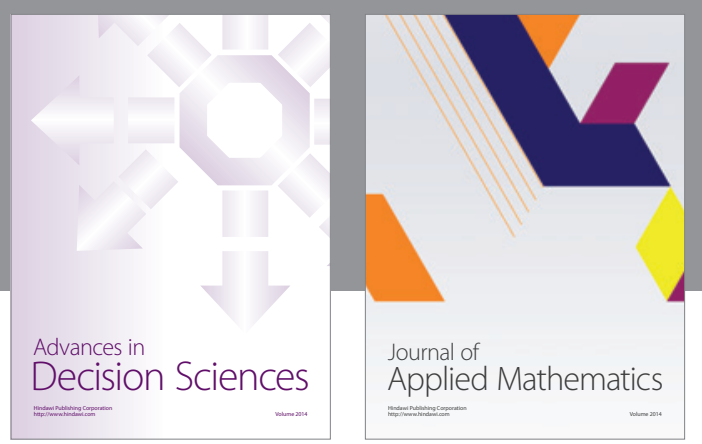

Algebra

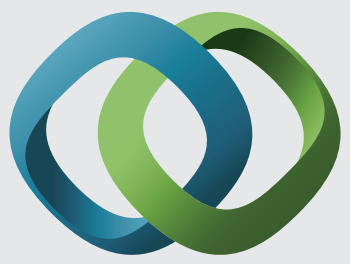

\section{Hindawi}

Submit your manuscripts at

https://www.hindawi.com
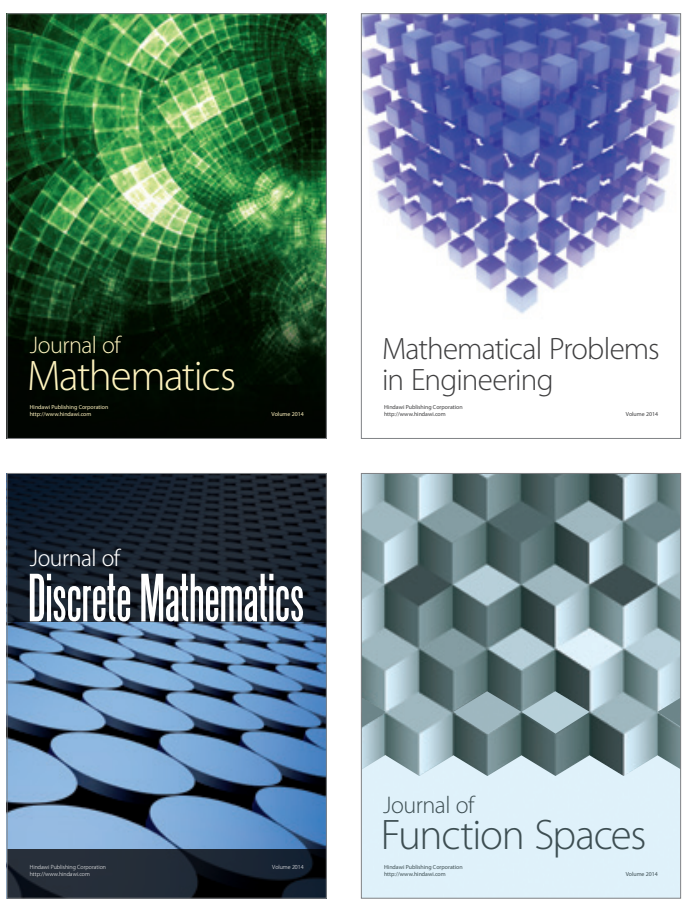

Mathematical Problems in Engineering
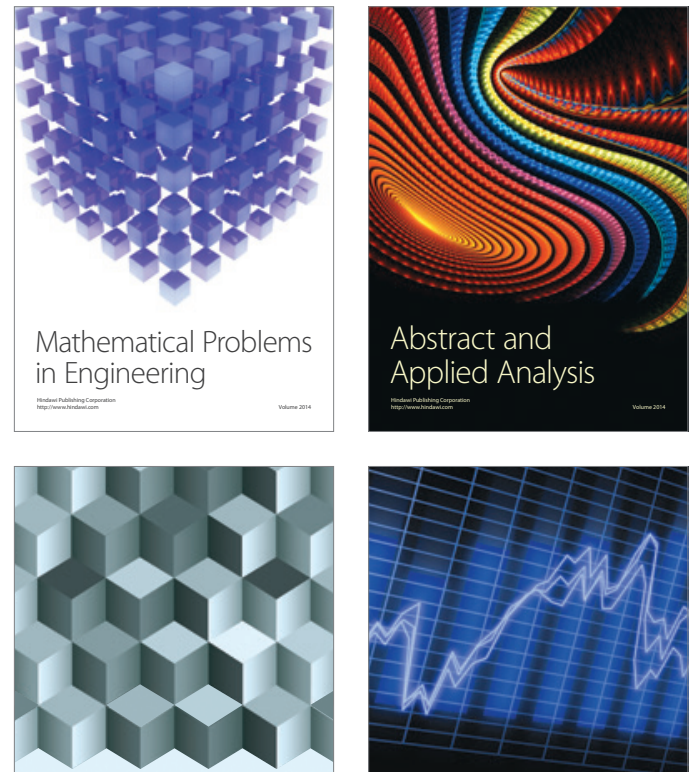

Journal of

Function Spaces

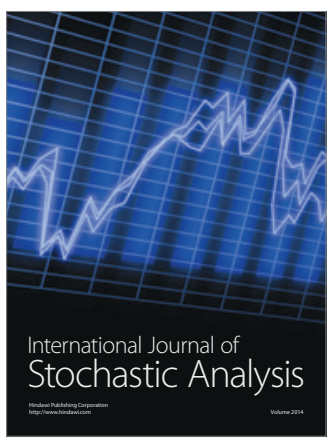

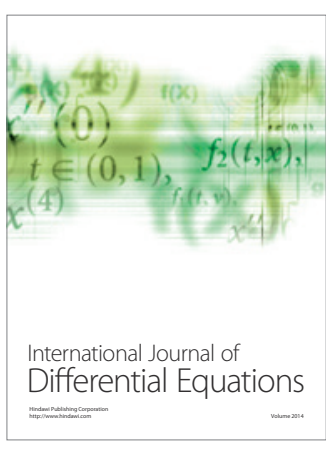
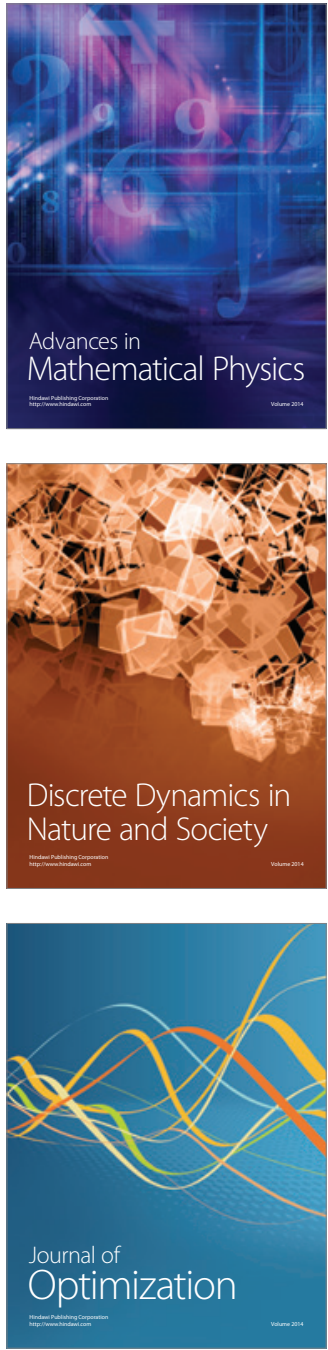